\title{
Characterisation of the in vitro activity of a Nitazoxanide- $N$-methyl- $1 H$-benzimidazole hybrid molecule against albendazole and nitazoxanide susceptible and resistant strains of Giardia intestinalis and its in vivo giardicidal activity
}

\author{
Félix Matadamas-Martínez'1,5, Benjamín Nogueda-Torres², Rafael Castillo', \\ Alicia Hernández-Campos ${ }^{1}$, María de la Luz Barrera-Valdes ${ }^{2,5}$, Gloria León-Ávila ${ }^{3}$, \\ José Manuel Hernández ${ }^{4}$, Lilián Yépez-Mulia ${ }^{5 /+}$
}

${ }^{1}$ Universidad Nacional Autónoma de México, Facultad de Química, Departamento de Farmacia, Mexico City, Mexico ${ }^{2}$ Escuela Nacional de Ciencias Biológicas-Instituto Politécnico Nacional, Departamento de Parasitología, Mexico City, Mexico ${ }^{3}$ Escuela Nacional de Ciencias Biológicas-Instituto Politécnico Nacional, Departamento de Zoología, Laboratorio de Genética, Mexico City, Mexico

${ }^{4}$ Centro de Investigación y de Estudios Avanzados del Instituto Politécnico Nacional, Departamento de Biología Celular, Mexico City, Mexico ${ }^{5}$ Instituto Mexicano del Seguro Social, Centro Médico Siglo XXI, Unidad de Investigación Médica en Enfermedades Infecciosas y Parasitarias, Hospital de Pediatría, Mexico City, Mexico

BACKGROUND It was previously demonstrated that CMC-20, a nitazoxanide and $N$-methyl-1H-benzimidazole hybrid molecule, had higher in vitro activity against Giardia intestinalis WB strain than metronidazole and albendazole and similar to nitazoxanide.

OBJETIVES To evaluate the in vitro activity of CMC-20 against $G$. intestinalis strains with different susceptibility/resistance to albendazole and nitazoxanide and evaluate its effect on the distribution of parasite cytoskeletal proteins and its in vivo giardicidal activity.

METHODS CMC-20 activity was tested against two isolates from patients with chronic and acute giardiasis, an experimentally induced albendazole resistant strain and a nitazoxanide resistant clinical isolate. CMC-20 effect on the distribution of parasite cytoskeletal proteins was analysed by indirect immunofluorescence and its activity was evaluated in a murine model of giardiasis.

FINDINGS CMC-20 showed broad activity against susceptible and resistant strains to albendazole and nitaxozanide. It affected the parasite microtubule reservoir and triggered the parasite encystation. In this process, alpha-7.2 giardin co-localised with CWP-1 protein. CMC-20 reduced the infection time and cyst load in feces of G. muris infected mice similar to albendazole.

MAIN CONCLUSIONS The in vitro and in vivo giardicidal activity of CMC-20 suggests its potential use in the treatment of giardiasis.

Key words: giardicidal activity - hybrid molecule - resistant strains - cytoskeletal proteins - in vivo activity

Giardia intestinalis is a flagellate protozoan that causes giardiasis, a diarrheal disease that affects human worldwide. G. intestinalis comprises eight different assemblages (A-H); from them, assemblages A and B are considered zoonotic since they infect both humans and domestic animals. Assemblage B is more commonly associated with human infections than assemblage A (58\% and $37 \%$, respectively). ${ }^{(1)}$ Giardia infection is an important cause of morbidity and due to its socioeconomic significance was included in the World Health Organization (WHO) Neglected Disease Initiative. ${ }^{(2)}$ It has been associated with intestinal dysbiosis and the subsequent development of chronic post-infection inflammatory bowel syndrome. ${ }^{(2)}$ In 2010, the World Health Organi-

doi: 10.1590/0074-02760190348

Financial support: Fondo de Investigación en Salud, IMSS

(grant FIS/IMSS/PROT/G17-2/1736).

FM-M was recipient of a fellowship from DGAPA UNAM.

+ Corresponding author: lilianyepez@yahoo.com

(D) https://orcid.org/0000-0001-8675-5671

Received 13 September 2019

Accepted 02 January 2020 zation (WHO) reported that Giardia caused 28.2 million cases of foodborne disease and 26,270 disabilityadjusted life years. ${ }^{(3)}$ In the United States, approximately 20,000 new cases of giardiasis are reported annually; however, from 2009 to 2010, the number of reported cases of giardiasis increased $1.9 \%$. $^{(4)}$ Importantly, migration from endemic places and an increase international travelling are changing the epidemiology of giardiasis in developed countries. In Sonora State (Norwest of Mexico) giardiasis prevalence between 20 to 30\% was determined in the childhood population. ${ }^{(5)}$ The ingestion of contaminated food and water with parasite cysts represents the main transmission route of giardiasis. Most infections in humans are asymptomatic, but in some cases acute or chronic disease are associated with severe diarrhea, malabsorption and weight loss. The trophozoite stage is the dividing and metabolically active form that is the main cause of the pathogenesis observed during giardiasis. The trophozoite adhesion to epithelial cells is an important step in the pathogenesis process and it is mainly mediated by the ventral disc. The ventral disc, median body, funis, eight motile flagella and axonemes, are constituted by the alpha and beta tubulin heterodimer, the structural unit of microtubules that together 
with giardins and actin microfilaments form the parasite cytoskeleton. The complex parasite cytoskeleton is essential for cell division, motility, attachment, intracellular transport and encystation/excystation process. ${ }^{(6)}$

Metronidazole (MTZ) is the drug of choice for the treatment of giardiasis, however, it causes severe secondary effects and treatment failure as well as parasite resistance have been reported.(7) Albendazole (ABZ), a benzimidazole derivative, is alternatively used in the treatment of giardiasis. It disrupts Giardia ventral disc organization. ${ }^{(8)} \mathrm{A}$ meta-analysis of the effectiveness of ABZ compared with MTZ as treatments for G. intestinalis infections showed that ABZ ( $400 \mathrm{mg} /$ day for five days) was as effective as MTZ. Importantly, patients treated with ABZ had fewer side effects in comparison to those treated with MTZ. ${ }^{(9)}$ Alternatively, nitazoxanide (NTZ), a 5-nitrothiazolyl derivative, is also used to treat giardiasis. In clinical trials, NTZ's efficacy varies between 71 to $100 \%$ and it is less toxic than MTZ. ${ }^{(10)}$ However, parasite resistance to NTZ and ABZ has been experimentally induced ${ }^{(11,12)}$ and it has been reported in clinical isolates. ${ }^{(13,14)}$ Therefore, efforts have been focused on the development of new giardicidal molecules. In this regard, Navarrete-Vazquez et al. ${ }^{(15)}$ synthesised a benzologue of NTZ against $G$. intestinalis $\left(\mathrm{IC}_{50}=0.297 \mu \mathrm{M}\right)$. It was four more active than NTZ $\left(\mathrm{IC}_{50}=1.214 \mu \mathrm{M}\right)$ and 18 times more active than MTZ $\left(\mathrm{IC}_{50}=5.35 \mu \mathrm{M}\right)$, respectively. Recently, we reported the synthesis and the in vitro giardicidal activity of a novel nitazoxanide and $N$-methyl-1H-benzimidazole hybrid molecule, named CMC-20. It showed against $G$. intestinalis WB strain an $\mathrm{IC}_{50}$ value of $0.010 \mu \mathrm{M}$, lower than the $\mathrm{IC}_{50}$ values of $\mathrm{ABZ}$ and MTZ and similar to NTZ. The proteomic analysis of CMC-20 treated trophozoites revealed significant changes in the expression level of alpha and beta tubulin, as well as some proteins that participate in the encystment process. ${ }^{(16)}$

Since CMC-20 showed potent activity against WB strain, a reference strain susceptible to ABZ and NTZ, our interest was to evaluate its giardicidal activity against parasite strains susceptible or resistant to these reference drugs. These strains included an isolate from a patient with chronic giardiasis and one from a patient with acute giardiasis; an experimentally induced ABZ resistant strain and a NTZ resistant clinical isolate. Besides, it was analysed by indirect immunofluorescence (IFI) the effect of CMC-20 on the distribution of cytoskeletal proteins such as beta tubulin and alpha tubulin, as well as acetylated, tyrosinated and detyrosinated-alpha tubulin and other cytoskeletal proteins such as alpha-7.2 giardin and actin. Finally, its giardicidal activity was determined in a murine model of giardiasis in comparison to ABZ.

\section{MATERIALS AND METHODS}

Chemistry - The benzimidazole derivative CMC-20 was prepared as previously described by our group. ${ }^{(16)}$ The chemical formula of CMC-20 is presented as Supplementary data.

Parasites - For susceptibility assays the following G. intestinalis strains were used: WB strain (ATCC \# 30957), a reference strain susceptible to ABZ and NTZ; isolates from patients with chronic (IMSS-1090-1) or acute (UNAM-0688-2) giardiasis, ${ }^{(17)}$ an experimentally induced ABZ resistant strain (BRIS/91/HEPU/1411) ${ }^{(18)}$ and a NTZ resistant clinical isolate (N1-INP). ${ }^{(13)}$ All the parasite strains were genotyped as assemblage AI.

Susceptibility assays - CMC-20, ABZ and NTZ at $26.1,37.6$ and $32.5 \mathrm{mM}$, respectively, were previously dissolved in dimethyl sulfoxide (DMSO) followed by drug dilution in Giardia culture medium, making a final stock solution of $26.1,37.6$ and $32.5 \mu \mathrm{M}$, respectively. Further dilutions were made in order to obtain the following working concentrations: $0.013-1.30 \mu \mathrm{M}$ of CMC-20; $0.018-1.88 \mu \mathrm{M}$ of ABZ and $0.016-1.62 \mu \mathrm{M}$ of NTZ. The maximum DMSO concentration in cultures was lower than $0.001 \%$, a concentration that does not affect $G$. intestinalis growth in vitro.

Parasites were cultured in TYI-S-33 modified medium supplemented with $10 \%$ calf serum and bovine bilis. Trophozoites $\left(5 \times 10^{4} / \mathrm{mL}\right)$ were incubated with the drugs at the different concentrations for $48 \mathrm{~h}$ at $37^{\circ} \mathrm{C}$. Trophozoites with DMSO (at the highest concentration used in working solutions) were included as negative control. At the end of the treatment trophozoites were washed and subcultured for another $48 \mathrm{~h}$ in fresh medium free of drugs. Parasites were counted with a hemocytometer and the concentration that inhibits the trophozoite growth by $50 \%\left(\mathrm{IC}_{50}\right)$ was calculated by Probit analysis. Three independent assays were performed by triplicate. For each assay, drugs were dissolved in DMSO and working solutions were newly prepared as already mentioned.

Cytotoxicity assay - Madin-Darby canine kidney cell line (MDCK) was used to assess the cytotoxic effect of ABZ and NTZ as previously described for CMC-20. (16) The cells were cultured in standard culture medium [MEM with $10 \%$ foetal bovine serum (FBS)]. The final concentration of DMSO in the culture medium remained below $0.01 \%$. The cultures were incubated with $\mathrm{ABZ}$ and NTZ at different concentrations for $24 \mathrm{~h}$ at $37^{\circ} \mathrm{C}, 5 \% \mathrm{CO}_{2}$ and $95 \%$ relative humidity. Untreated cells were included as controls. Cytotoxicity was determined using the colorimetric MTT assay. Viability percentage was calculated with respect to control cells and 50\% cytotoxicity concentration $\left(\mathrm{CC}_{50}\right)$ was estimated using the GraphPad Prism (4.0 version). The assay was done by triplicate.

Production of antibodies - Antibodies against recombinant actin and alpha-7.2 giardin proteins were produced in $\mathrm{BALB} / \mathrm{c}$ mice meanwhile antibodies against CWP-1 were produced in Wistar rats. Animals were injected intraperitoneally with $100 \mu \mathrm{g}$ recombinant proteins at days 0,15 and 30 . The first boost was done with 10:1 protein/ Complete Freund's adjuvant mixture (Sigma-Aldrich), and the following boosts were done with 1:1 protein/Incomplete Freund's adjuvant mixture, (Sigma-Aldrich). Immune sera were obtained four days after the last boost and assayed by enzyme-linked immunosorbent assay (ELISA).

IFI assays - For this purpose, trophozoites of G. intestinalis WB strain $\left(2 \times 10^{6}\right)$ were incubated for $24 \mathrm{~h}$ in culture medium with CMC-20 at the same concen- 
tration $(7.8 \mu \mathrm{M})$ previously used for proteomic studies. (16) Trophozoites without treatment were included as negative control. Control and CMC-20 treated parasites were placed on poly-L-lysine coated glass slides (Sigma-Aldrich) and incubated for $10 \mathrm{~min}$ to allow cells to attach to the glass surface. Then, parasites were fixed with 4\% paraformaldehyde in phosphate-buffered saline (PBS) $\mathrm{pH} 7.2$ for $15 \mathrm{~min}$ and washed twice with PBS. The parasites were permeabilised with $0.5 \%$ Triton X100 -SDS for $10 \mathrm{~min}$ and washed twice with PBS, and nonspecific binding was blocked with $1 \%$ bovine serum albumin (BSA) in PBS for $1 \mathrm{~h}$ at room temperature. After this, parasites were incubated at room temperature for $1 \mathrm{~h}$ with the following monoclonal antibodies: anti-alpha tubulin clone B-512 (Sigma-Aldrich) (1:1000), anti- acetylated tubulin clone 6-11B-1 (Sigma-Aldrich) (1:1000), anti-tyrosinated tubulin clone TUB-1A2 (Sigma-Aldrich) (1:100), anti-detyrosinated tubulin clone YOL1/34 (Millipore)(1:100), anti-beta tubulin clone KMX-1(Millipore) (1:500), and polyclonal antibodies against either alpha-7.2 giardin (1:1000), actin (1:500) or CWP-1 (1:1000). Secondary antibodies anti-mouseFITC and anti-rat-TRITC (Millipore) (1:500) were incubated for $1 \mathrm{~h}$ at room temperature. The cover slips were washed three times in PBS and mounted on glass slides with Vectashield (Vector Laboratories). The images were obtained using a Leica confocal microscope and epifluorescence (Leica DMIRE2 and Olympus).

Evaluation of the in vivo giardicidal activity of CMC-20 - The biological activity of CMC-20 was evaluated against G. muris infection in BALB/c mice. ABZ was included as the reference drug. For this, groups of 6 female BALB/c mice aged four-six weeks old were each orally infected with $1 \times 10^{3}$ cysts of G. muris suspended in $0.2 \mathrm{~mL}$ PBS, pH 7.2. CMC-20 and ABZ at $50 \mathrm{mg} /$ $\mathrm{kg}$ /day were administered intragastrically at days seven and eight post-infection (pi). Animals infected but not treated were included as negative control. Feces excreted from control group and treated groups were collected for over a $2 \mathrm{~h}$ period daily from tree to 35 day after infection. Cysts were purified from feces using a sucrose gradient and the number of shed cysts was counted using a hemocytometer. Animal experiments were performed according to the Norma Oficial Mexicana (NOM062-Z00-1999) published on August 22, 2009.

\section{RESULTS}

Activity of CMC-20 against $G$. intestinalis strains The activity of CMC-20 and the reference drugs, ABZ and NTZ, against all the parasite stains tested is presented in Table. ABZ was active against WB strain and isolates from patients with chronic (IMSS-1090-1) or acute (UNAM-0688-2) giardiasis $\left(\mathrm{IC}_{50}\right.$ of $=0.047,0.040$ and $0.034 \mu \mathrm{M}$, respectively), however, it was more active against the isolate from a patient with acute giardiasis. NTZ was more active against both isolates $\left(\mathrm{IC}_{50}\right.$ $=0.009 \mu \mathrm{M})$ than against WB strain $\left(\mathrm{IC}_{50}=0.018 \mu \mathrm{M}\right)$. In relation to CMC-20, it was active against the isolates and WB strain, however, it was more active against WB strain $\left(\mathrm{IC}_{50}\right.$ of $\left.0.015 \mu \mathrm{M}\right)$ than against the isolates from patients with chronic and acute giardiasis $\left(\mathrm{IC}_{50}=0.052\right.$ and $0.033 \mu \mathrm{M}$, respectively). CMC-20 was 1.6 times more active against the acute giardiasis isolate in comparison to the chronic giardiasis isolate. In general, NTZ was 5.8 and 3.7 times more active than CMC-20 and 4.4 and 3.8 times more active than ABZ against the isolates from patients with chronic and acute giardiasis. However, CMC-20 was three times more active than ABZ against WB strain $\left(\mathrm{IC}_{50}=0.015 \mu \mathrm{M}\right.$ vs $\left.0.047 \mu \mathrm{M}\right)$, but similar to NTZ $\left(\mathrm{IC}_{50}=0.015 \mu \mathrm{M}\right.$ vs $\left.0.018 \mu \mathrm{M}\right)$.

In relation to the ABZ resistant strain, CMC-20 was three times more potent than $\mathrm{ABZ}\left(\mathrm{IC}_{50}=0.045 \mu \mathrm{M}\right.$ vs $0.137 \mu \mathrm{M})$; but showed half the activity of NTZ $\left(\mathrm{IC}_{50}=\right.$ $0.045 \mu \mathrm{M}$ vs $0.021 \mu \mathrm{M}$ ). Regarding the NTZ resistant clinical isolate, CMC-20 was 28 times more active than $\mathrm{NTZ}\left(\mathrm{IC}_{50}=0.071\right.$ vs $\left.1.97 \mu \mathrm{M}\right)$ and 3.9 times more active than $\mathrm{ABZ}\left(\mathrm{IC}_{50}=0.071\right.$ vs $\left.0.277 \mu \mathrm{M}\right)$.

Cytotoxicity assays - The cytotoxicity of CMC-20, ABZ and NTZ was tested on Madin-Darby canine kidney cell line (MDCK) and their $\mathrm{CC}_{50}$ values were $>26 \mu \mathrm{M},{ }^{(16)}$ $>37.6 \mu \mathrm{M}$ and $>32.5 \mu \mathrm{M}$, respectively. To calculate their selectivity index (SI) against the parasite strains, $\mathrm{CC}_{50}$ values of $26 \mu \mathrm{M}, 37.6 \mu \mathrm{M}$ and $32.5 \mu \mathrm{M}$ of CMC-20, $\mathrm{ABZ}$ and NTZ, respectively, were used (Table). NTZ had the

TABLE

Activity $\left(\mathrm{IC}_{50} \mu \mathrm{M}\right)$ of Albendazole (ABZ), nitazoxanide (NTZ) and CMC-20 against susceptible and resistant strains of Giardia intestinalis and selectivity index* (SI)

\begin{tabular}{lccccc}
\hline Compound & WB strain & IMSS-1090-1 isolate & UNAM-0688-2 isolate & rABZ strain & rNTZ isolate \\
\hline ABZ & $0.047 \pm 0.001$ & $0.040 \pm 0.004$ & $0.034 \pm 0.003$ & $0.137 \pm 0.01$ & $0.277 \pm 0.001$ \\
SI & 800 & 940 & 1105 & 274 & 136 \\
\hline NTZ & $0.018 \pm 0.005$ & $0.009 \pm 0.005$ & $0.009 \pm 0.005$ & $0.021 \pm 0.007$ & $1.973 \pm 0.2$ \\
SI & 1805 & 3611 & 3611 & 1547 & 16 \\
CMC-20 & $0.015 \pm 0.002$ & $0.052 \pm 0.003$ & $0.033 \pm 0.004$ & $0.045 \pm 0.004$ & $0.071 \pm 0.004$ \\
SI & 1733 & 500 & 787 & 577 & 366
\end{tabular}

rABZ: an induced albendazole resistant strain; rNTZ: nitazoxanide resistant clinical isolate. *The selectivity index (SI) of CMC-20, $\mathrm{ABZ}$ and NTZ was calculated as the ratio of cytotoxicity to biological activity $\left(\mathrm{SI}=\mathrm{CC}_{50} \mathrm{MDCK}\right.$ cells/IC $\mathrm{C}_{50}$ strains). 
highest SI against the clinical isolates and the $\mathrm{ABZ}$ resistant strain. However, the SI of CMC-20 was higher than NTZ against the NTZ resistant clinical isolate and its SI against WB strain was close to NTZ. In addition, CMC20 showed higher SI than ABZ against WB strain and the $\mathrm{ABZ}$ and NTZ resistant isolates.

Analysis of the effect of $\mathbf{C M C}-\mathbf{2 0}$ on the distribution of cytoskeletal and encystation proteins by IFI - In this study the effect of CMC-20 on the distribution of different cytoskeletal parasite proteins was analysed by IFI. These assays showed the presence of alpha tubulin and acetylated-alpha tubulin in the ventral disc, flagella, basal bodies and median body of control WB trophozoites (Fig. 1A, C). In CMC-20 treated parasites, alpha tubulin and acetylated-alpha tubulin were recognised in the same structures as in control, with the exception of the median body. In addition, major morphological changes and some rounded cells with retraction of the flagella were observed (Fig. 1B, D). The presence of tyrosinated-alpha tubulin, using the antibody TUB-1A2, was observed in the cytoplasm, flagella and median body of control parasites (Fig. 1E). In CMC-20 treated parasites, tyrosinated-alpha tubulin was recognised in the same structures as in control with the exception of the median body (Fig. 1F). However, no recognition of detyrosinated-alpha tubulin was observed in both control and treated parasites.

Regarding beta tubulin, the flagella and median body of control parasites were recognised using the antibody KMX-1 (Fig. 1G). However, the median body was not stained for beta tubulin in CMC-20 treated parasites (Fig. 1H). The no recognition of the median body by antibodies anti-alpha and anti- beta tubulin was observed in $80 \%$ of the treated cells. Alpha-7.2 giardin was localised in association with the plasma membrane, ventral disc and slightly on flagella of control parasites (Fig. 2A). Nevertheless, in CMC-20 treated parasites, alpha-7.2 giardin was evidenced on the surface of cyst-like cells as well as in vesicle-like structures (Fig. 2B, C). The use of an antiCWP-1 antibody confirmed that the rounded cells were encysted parasites and the vesicle-like structures were encystation specific vesicles (ESVs) (Fig. 2E, F). About 10\% of the treated trophozoites were transformed into cysts. Control parasites did not stain for CWP-1 (Fig. 2D, G). Interestingly, it was evident that in CMC-20 treated parasites, CWP-1 co-localised with alpha-7.2 giardin (Fig. $2 \mathrm{H}$, I). Actin was detected in the cortex, flagella and around nuclei of control trophozoites (Fig. 3A), meanwhile in treated parasites, the presence of actin was observed in patches and it was also detected on the surface of cysts (Fig. 3B, C). It is worth mentioning that actin co-localised with CWP-1 in some ESVs of treated parasites (Fig. 3H) and on the surface of cysts (Fig. 3I). Control parasites did not stain for CWP-1 (Fig. 3D, G).

Evaluation of the in vivo giardicidal activity of CMC-20 - The giardicidal activity of CMC-20 was evaluated in BALB/c mice experimentally infected with G. muris. CMC-20 at $50 \mathrm{mg} / \mathrm{kg} /$ day was administered intragastrically at days seven and eight pi. Non-treated infected mice and infected mice treated with ABZ (50 $\mathrm{mg} / \mathrm{kg} /$ day) were included as controls (Fig. 4). The release of cysts in feces of non-treated infected mice (Fig. 4 -•-) was observed till day 29 pi, however, intermittent release was detected from day 22 till day 28 pi. No cysts release was observed from day 29 to $35 \mathrm{pi}$. On the other hand, in infected animals treated with CMC-20 (Fig. 4 - $\boldsymbol{m - )}$, the number of cysts released in feces was drastically reduced as well as the infection time in comparison to the infection control. Cyst load was reduced immediately after the first administration of CMC-20, decreas-
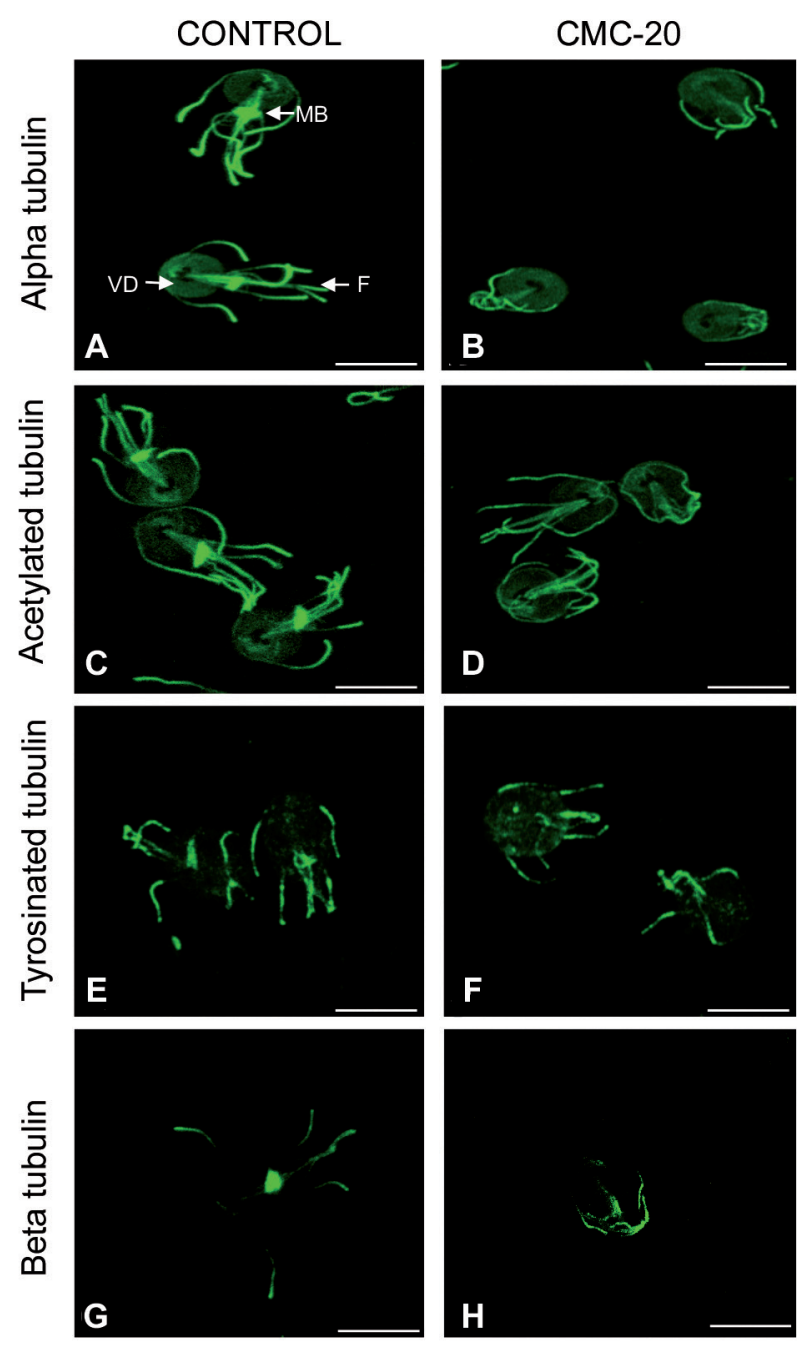

Fig. 1: distribution of parasite cytoskeletal proteins in control (A,C,E,G) and CMC-20 treated trophozoites (B,D,F,H) analysed by indirect immunofluorescence (IFI) using a set of specific antibodies against cytoskeletal proteins. In control parasites the presence of alpha tubulin (A) and acetylated-alpha tubulin (C) was observed mainly in the ventral disc, flagella and median body. Tyrosinated-alpha tubulin (E) was localised in the cytoplasm, flagella and in the median body of control parasites. In addition, beta tubulin $(\mathrm{G})$ was observed in the flagella and in the median body of control parasites. In CMC20 treated parasites, cytoskeletal proteins were localised in the same structures as in control parasites, with the exception of median body. Trophozoites treated with CMC-20 lose their characteristic pear shape and in some cells flagella retraction was observed (VD: ventral disc; MB: median body; F: flagella; Bar: $5 \mu \mathrm{m})$. 

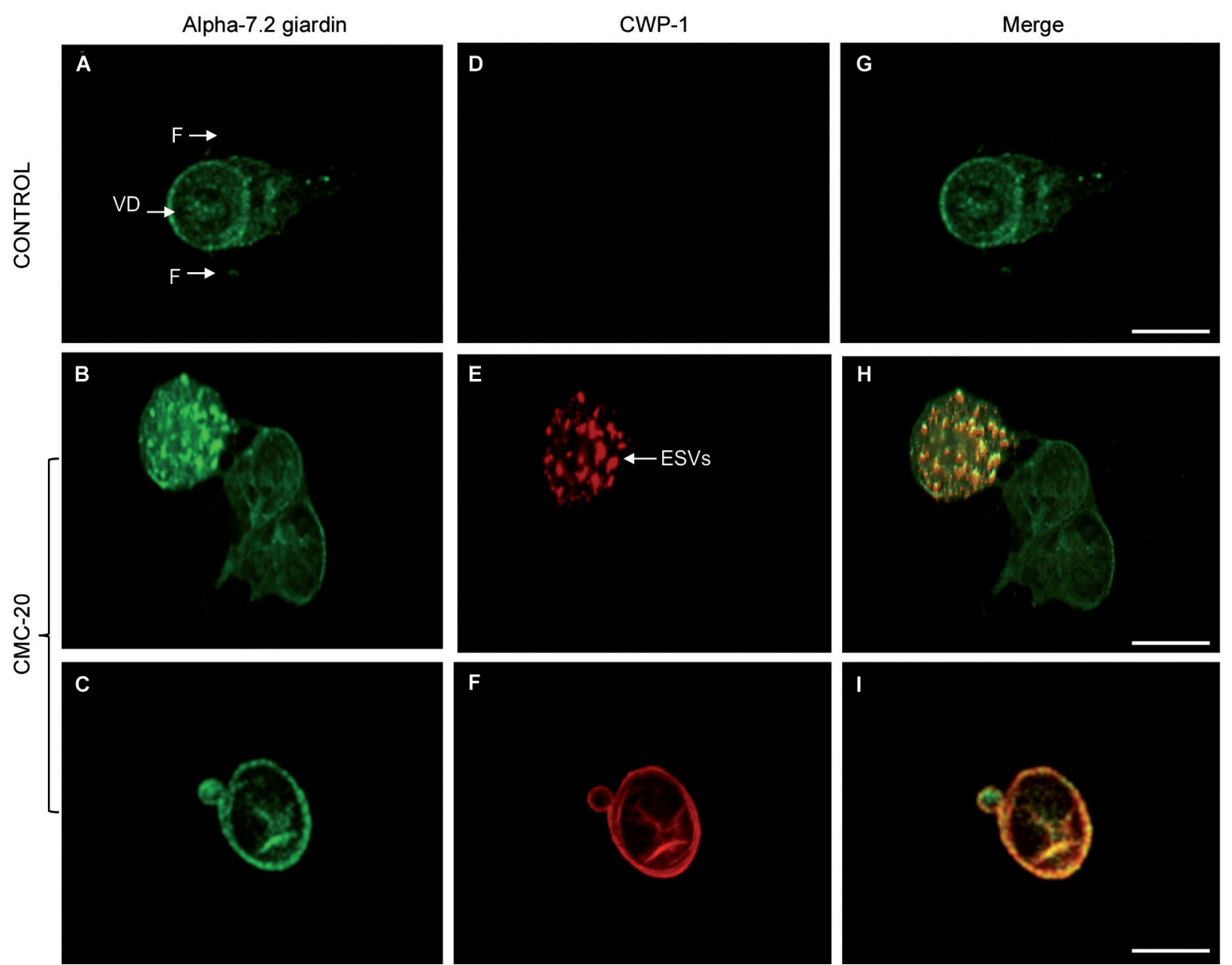

Fig. 2: localisation of alpha-7.2 giardin and CWP-1 in control and CMC-20 treated parasites. Alpha-7.2 giardin was localised in association with the plasma membrane, ventral disc and slightly on flagella of control trophozoites (A, G), however, it was observed in vesicle-like structures (B) and on the surface of CMC-20 treated parasites (C). Control parasites did not stain for CWP-1 (D). CWP-1 localised in ESVs and on the surface of CMC-20 treated parasites (E, F). Alpha-7.2 giardin co-localised with CWP-1 both in ESVs and on the cell surface of treated parasites (H and I). VD: ventral disc; F: flagella; ESVs: encystation specific vesicles; Bar: $5 \mu \mathrm{m}$.

ing from 16473 cysts at day seven pi to 4627 cysts at day eight pi. At day 15 pi no cysts were detected, however, at day 16 and 21 pi an intermittent release of cysts was observed, reaching a maximum release of 370 cysts. From day 22 pi until day 35 pi no cyst release was detected. In infected mice treated with ABZ (Fig. 4 - $\mathbf{\Delta}$-), the release of cysts started to decrease after day 8 pi (28877 cysts) and no cysts in feces were detected at day $17 \mathrm{pi}$, however, at day 18 and 20 pi an intermittent release of cysts was observed (185 and 370 cysts, respectively). Cyst release was not detected from day 21 pi up to day 35 pi.

\section{DISCUSSION}

There are few different classes of drugs available for treatment of human giardiasis, and therapeutic failure occurs frequently, being drug resistance the most important cause. Metronidazole, a nitroimidazole derivative, is commonly used for first-line treatment of human giardiasis; however, cases of drug-resistance have been frequently reported.(7) A prevalence of metronidazoleresistant clinical isolates was up to $20 \%$ in people at- tended in a travel clinic in Spain, especially those with infections acquired in Asia. ${ }^{(19)}$ Besides, metronidazole treatment failure increased from $15.1 \%$ in 2008 to $40.2 \%$ in 2013 in London, England. ${ }^{(7)}$ ABZ, a benzimidazole derivative, and NTZ, a nitrothiazole derivative, are alternatively used in the treatment of giardiasis. Regarding ABZ, treatment failure has also been reported ${ }^{(20)}$ and $\mathrm{ABZ}$ resistance has been described in clinical isolates. (14) In relation to NTZ, clinical cure was demonstrated in only $50 \%$ of nitroimidazole refractory cases treated with $\mathrm{NTZ}^{(7)}$ and more recently, a case of treatment failure with NTZ was reported; and NTZ resistance was confirmed in the clinical isolate (N1-INP). ${ }^{(13)}$ Therefore, development of new giardicidal drugs remains urgent, in particular given the disadvantages of the available drugs mentioned above.

Then, in this study it was of interest to investigate the activity of CMC-20, a hybrid molecule of NTZ and $\mathrm{N}$-methyl-1H-benzimidazole, against $G$. intestinalis strains with different susceptibility or resistance to the reference drugs, ABZ and NTZ. Data obtained showed 

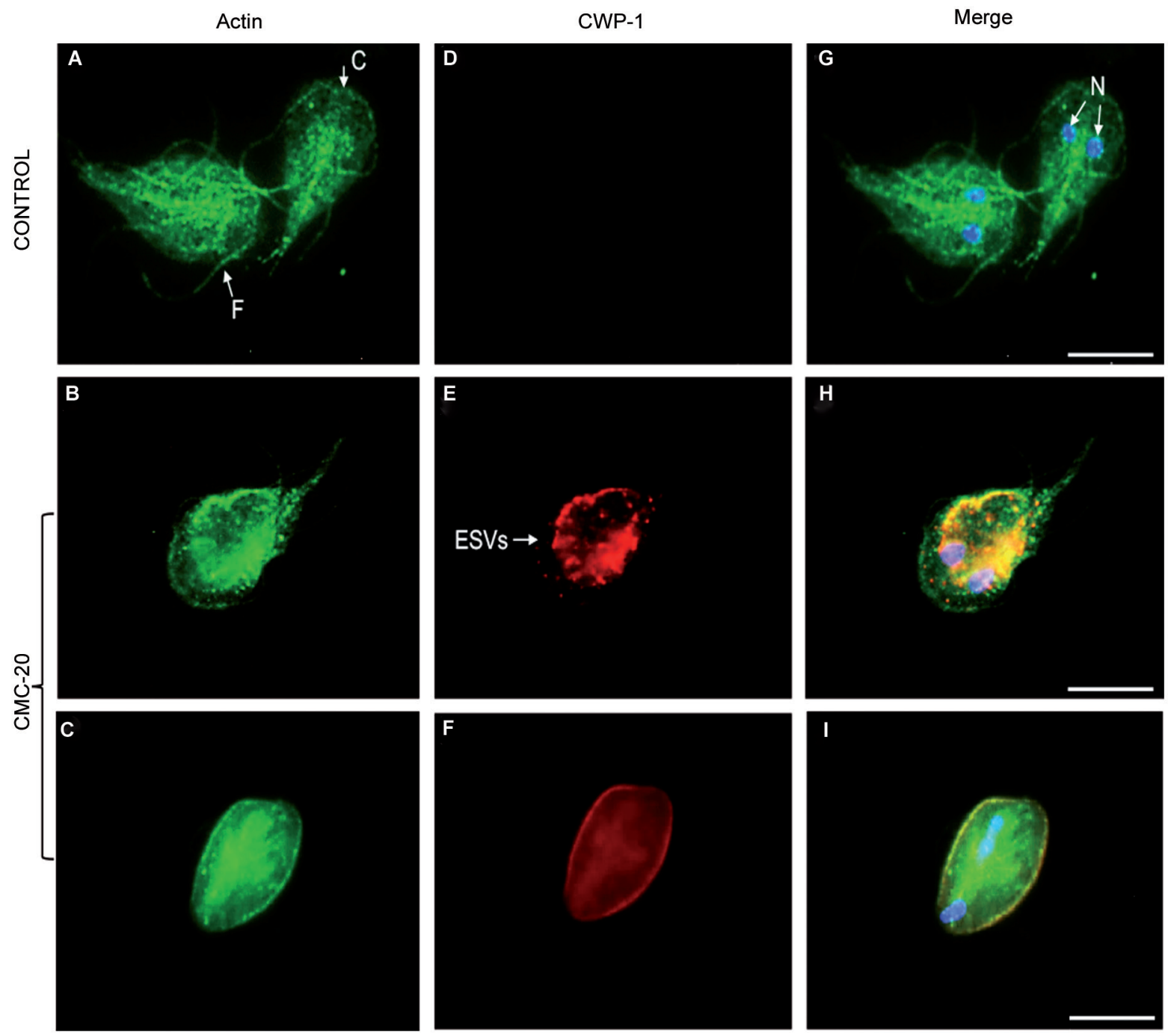

Fig. 3: actin localisation in control and CMC-20 treated parasites. Actin localised to the cortex, flagella and around nuclei of control parasites (A, G). Control trophozoites did not stain for CWP-1 (D). In CMC-20 treated parasites actin was detected in the caudal flagella, the cortex and as condensed patches in the cytoplasm (B). It was also detected on the surface of cysts (C). CWP-1 was detected in ESVs (E) and on the surface of cysts (F). Co-localisation of CWP-1 with actin was observed (H, I). The nuclei are stained with DAPI (blue). F: flagella; C: cortex; N: nuclei; Bar: $5 \mu \mathrm{m}$.

that NTZ was more active than CMC-20 and showed the highest SI against the chronic and acute clinical isolates as well as the ABZ resistant strain. Nevertheless, CMC20 showed $\mathrm{IC}_{50}$ values in the nanomolar order against all tested strains; and importantly, it showed 3 and 28 times higher activity against ABZ and NTZ resistant strains than ABZ and NTZ, respectively. Besides, CMC-20 showed two and 23 times higher selectivity against the ABZ and NTZ resistant strains than ABZ and NTZ, respectively. It is important to point out that, although NTZ was highly active and selective against parasite strains susceptible to this drug, resistance to NTZ as well as ABZ has been reported not only on experimentally induced resistant strains, ${ }^{(11,12)}$ but also in clinical isolates ${ }^{(13,14)}$ and treatment failure has been documented. ${ }^{(7,20)}$ In this regard, CMC-20 could have a potential use in the treatment of giardiasis.

Previous proteomic analysis of $G$. intestinalis WB strain treated with CMC-20 revealed changes on the expression of alpha and beta tubulin as well as some proteins involved in the encystment process, ${ }^{(16)}$ but it was not further characterised. Herein, IFI studies, demonstrated that CMC-20 induced changes not only on the distribution of the cytoskeletal proteins, alpha and beta tubulin, but also of the post-translationally modified acetylated and tyrosinated-alpha tubulin. These cytoskeletal proteins were localised, in agreement with other studies, ${ }^{(21,22)}$ in structures such as the ventral disc, flagella, median body and basal bodies of the control parasites. Significantly, these proteins were not detected in the median body of around $80 \%$ of the CMC-20 treated trophozoites. The median body is considered to be a microtubule reservoir that participates in the ventral disc progenesis. ${ }^{(21)}$ Interestingly, previous FESEM studies performed after membrane detergent extraction of CMC-20 treated parasites showed the ventral disc spiral disorganised and the bare area expanded. Besides, proteomic analysis demonstrated that CMC-20 down- 


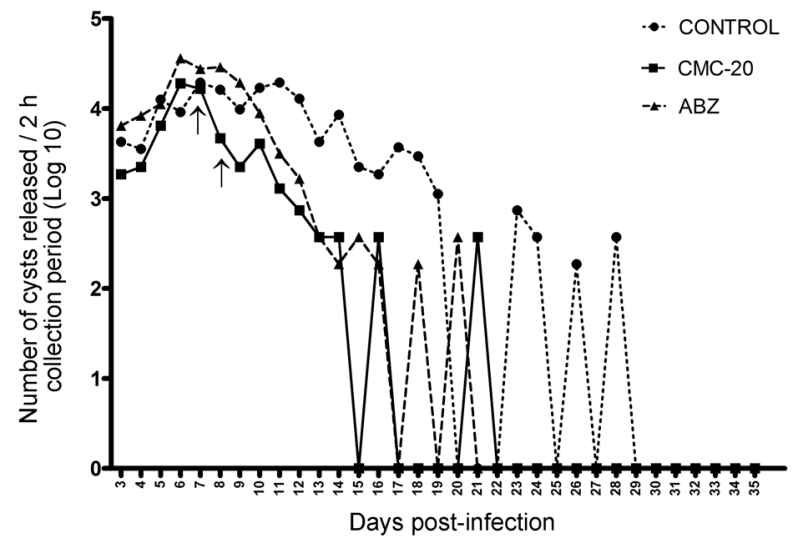

Fig. 4: number of cysts released in the feces of non-treated Giardia muris infected mice or infected mice treated with CMC-20 or albendazole (ABZ). BALB/c mice were orally infected with $1 \times 10^{3}$ G. muris cysts and treated with CMC-20 or ABZ at $50 \mathrm{mg} / \mathrm{kg} /$ day at days 7 and 8 after infection (arrows). Each point represents the number of cysts $\left(\log _{10}\right)$ released per $2 \mathrm{~h}$ collection period of six animals from days 3 to $35 \mathrm{pi}$.

regulated the expression of alpha and beta tubulin. ${ }^{(16)}$ These observations together with the absence of the median body in treated parasites, observed by IFI, indicate that CMC-20 affects the parasite cytoskeletal proteins. The absence of the median body has also been shown in Giardia trophozoites treated with drugs that affect the parasite microtubules, such as ABZ and nocodazole. ${ }^{(8,23)}$

On the other hand, the presence of tyrosinated-alpha tubulin but not detyrosinated- alpha tubulin, can be explained based on the examination of the Giardia Genome Project. ${ }^{(24)}$ It revealed the presence of a gene (Contig 725, ORF 14498) with a high degree of similarity to other tubulin tyrosine ligase family genes, and the predicted protein sequence contains a canonical tubulin tyrosine ligase, However, the examination of $\mathrm{C}$ terminal fragments of alpha tubulin showed complete retention of the terminal tyrosine indicative of the absence of a tubulin carboxypeptidase. ${ }^{(24)}$ In addition, it is known that acetylated-alpha tubulin forms more stable microtubules, meanwhile tyrosinated-alpha tubulin is present in more dynamic microtubules, then, G. intestinalis cytoskeleton seems to be constituted by both types of microtubules.

The presence of CWP-1 in ESVs and on the surface of CMC-20 treated parasites was shown by IFI assays, confirming that CMC-20 triggers the parasite encystation. It is worth mentioning that although CMC-20 induces some cells to encyst (around 10\%), previous FESEM studies of parasites treated with CMC-20 at 0.010 $\mu \mathrm{M}$ and $7.8 \mu \mathrm{M}$ showed the cysts surface severely damaged, ${ }^{(16)}$ suggesting that they may be unviable. It may be that the stress conditions induced by CMC-20, severely affect the trophozoite survival, but only some of them can undergo the encystment process, although the cysts surface is damaged. ABZ and some benzimidazole derivatives also induce the encystment process in Giardia. ${ }^{(25)}$

The localisation of alpha-7.2 giardin associated to the plasma membrane of trophozoites was also shown by Weiland et al. ${ }^{(26)}$ In this regard, the association of several alpha-giardins (alpha-1, alpha -2, alpha-7.3, alpha -8 and alpha-11) to the plasma membrane has been demonstrated. ${ }^{(26,27)}$ It was proposed that alpha giardins may stabilise the cytoskeleton by crosslinking the plasma membrane with the parasite microtubules. ${ }^{(26)}$ For the first time, the co-localisation of CWP-1 with alpha-7.2 giardin in ESVs and on the surface of parasites during the encystment process was demonstrated. In previous studies of lowdensity ESVs fractions, the presence of alpha-7 giardin was detected by two-dimensional electrophoresis and mass spectrometry analysis, however, it was considered as a contamination during ESVs purification. ${ }^{(28)}$ The association of alpha-7.2 giardin with CWP-1, during parasite encystation, requires further studies. Moreover, the colocalisation of actin with CWP-1 in some ESVs was also observed. In this regard, Paredez et al. ${ }^{(29)}$ described the recruitment of actin to mature ESVs during the encystment process and Castillo-Romero et al. ${ }^{(30)}$ demonstrated that Rab11 and actin are involved in Giardia encystation.

The in vivo giardicidal activity of CMC-20 was shown in an experimental model of giardiasis. CMC-20 induced an important reduction in the cyst load as well as in the infection time in comparison to non-treated infected mice. It is worth to mention that the giardicidal activity of CMC-20 was similar to that shown by ABZ.

Herein, the broad activity and high selectivity of CMC-20 against isolates from patients with chronic or acute giardiasis as well as ABZ and NTZ resistant strains was demonstrated. Indeed, the effect of CMC-20 on the parasite microtubule reservoir, demonstrated by IFI assays, contributes to its anti-Giardia activity. The co-localisation of alpha-7.2 giardin and actin with CWP-1 during the encystment process induced by CMC-20 needs further studies. CMC-20 reduced the infection time and cyst load in feces of Giardia infected mice similar to ABZ. Further pharmacokinetic and toxicological studies, among others are required in order to confirm the potential use of CMC-20 in the treatment of giardiasis.

\section{ACKNOWLEDGEMENTS}

To Jaime Escobar (Centro de Investigación y Estudios Avanzados, IPN, Mexico) for his technical support in Confocal Microscopy. NTZ resistant clinical isolate was donated by Dr Gabriel López-Velázquez and Dr Ignacio de la Mora (Instituto Nacional de Pediatría, Mexico). IMSS-1090-1, UNAM-0688-2 and BRIS/91/HEPU/1411 isolates were provided by Dr Roberto Cedillo-Rivera (Universidad Autónoma de Yucatán, Mexico).

\section{AUTHORS' CONTRIBUTION}

LY-M - Conceptualisation; LY-M, RC and NT-B - formal analysis; LY-M - funding acquisition; FM-M, BV-ML and NT-B - methodology; LY-M - project administration; AHC, LA-G and H-JM - supervision; FM-M, LY-M, LA-G and H-JM - writing and editing. The authors declare that they have no conflict of interest.

\section{REFERENCES}

1. Ryan U, Cacciò SM. Zoonotic potential of Giardia. Int J Parasitol. 2013; 43(12-13): 943-56.

2. Beatty JK, Akierman SV, Motta JP, Muise S, Workentine ML, Harrison JJ, et al. Giardia duodenalis induces pathogenic dysbiosis of human intestinal microbiota biofilms. Int J Parasitol. 2017; 47(6): 311-26. 
3. Havelaar AH, Kirk MD, Torgerson PR, Gibb HJ, Hald T, Lake RJ, et al. World Health Organization Global estimates and regional Comparisons of the burden of foodborne disease in 2010. PLoS Med. 2015; 12(12): 1-23.

4. Yoder JS, Gargano JW, Wallace RM, Beach MJ. Centers for Disease Control and Prevention (CDC). Giardiasis surveillance - United States, 2009-2010. MMWR Surveill Summ. 2012; 61(5): 13-23.

5. Quihui-Cota L, Morales-Figueroa GG, Javalera-Duarte A, PonceMartínez JA, Valbuena-Gregorio E, López-Mata MA. Prevalence and associated risk factors for Giardia and Cryptosporidium infections among children of northwest Mexico: a cross-sectional study. BMC Public Health. 2017; 17: 1-10.

6. Elmendorf HG, Dawson SC, McCaffery JM. The cytoskeleton of Giardia lamblia. Int J Parasitol. 2003; 33: 3-28.

7. Nabarro LE, Lever RA, Armstrong M, Chiodini PL. Increased incidence of nitroimidazole-refractory giardiasis at the Hospital for Tropical Diseases, London, 2008-2013. Clin Microbiol Infect. 2015; 21: 791-6.

8. Chávez B, Cedillo-Rivera R, Martínez-Palomo A. Giardia lamblia: ultrastructural study of the in vitro effect of benzimidazoles. J Protozool. 1992; 39: 510-15.

9. Solaymani-Mohammadi S, Genkinger JM, Loffredo CA, Singer SM. A meta-analysis of the effectiveness of albendazole compared with metronidazole as treatments for infections with Giardia duodenalis. PLoS Negl Trop Dis. 2010; 4(5): 1-9.

10. Romero-Cabello R, Guerrero LR, Muñoz-García MR, GeyneCruz A. Nitazoxanide for the treatment of intestinal protozoan and helminthic infections in Mexico. Trans R Soc Trop Med Hyg. 1997; 91: 701-03.

11. Müller J, Ley S, Felger I, Hemphill A, Müller N. Identification of differentially expressed genes in a Giardia lamblia WB C6 clone resistant to nitazoxanide and metronidazole. J Antimicrob Chemother. 2008; 62: 72-82.

12. Martínez-Espinosa R, Argüello-García R, Saavedra E, OrtegaPierres G. Albendazole induces oxidative stress and DNA damage in the parasitic protozoan Giardia duodenalis. Front Microbiol. 2015; 6: 1-14

13. Reyes-Vivas H, de la Mora I, Castillo-Villanueva A, Yépez-Mulia L, Hernández-Alcántara G, Figueroa-Salazar R, et al. Giardial triosephosphate isomerase as possible target of the cytotoxic effect of omeprazole in Giardia lamblia. Antimicrob Agents Chemother. 2014; 58(12): 7072-82.

14. Lemée V, Zaharia I, Nevez G, Rabodonirina M, Brasseur P, Ballet JJ, et al. Metronidazole and albendazole susceptibility of 11 clinical isolates of Giardia duodenalis from France. J Antimicrob Chemother. 2000; 46(5): 819-21.

15. Navarrete-Vazquez G, Chávez-Silva F, Argotte-Ramos R, Rodríguez-Gutiérrez MC, Chan-Bacab MJ, Cedillo-Rivera R, et al. Synthesis of benzologues of nitazoxanide and tizoxanide: a comparative study of their in vitro broad-spectrum antiprotozoal activity. Bioorg Med Chem Lett. 2011; 21(10): 3168-71.
16. Matadamas-Martínez F, Castillo R, Hernández-Campos A, Méndez-Cuesta C, de Souza W, Gadelha AP, et al. Proteomic and ultrastructural analysis of the effect of a new nitazoxanide-Nmethyl-1H-benzimidazole hybrid against Giardia intestinalis. Res Vet Sci. 2016; 105: 171-9.

17. Cedillo-Rivera R, Darby JM, Enciso-Moreno JA, Ortega-Pierres G, Ey PL. Genetic homogeneity of axenic isolates of Giardia intestinalis derived from acute and chronically infected individuals in Mexico. Parasitol Res. 2003; 90(2): 119-23.

18. Upcroft J, Mitchell R, Chen N, Upcroft P. Albendazole resistance in Giardia is correlated with cytoskeletal changes but not with a mutation at amino acid 200 in beta-tubulin. Microb Drug Resist. 1996; 2(3): 303-8.

19. Muñoz Gutiérrez J, Aldasoro E, Requena A, Comin AM, Pinazo MJ, Bardají A, et al. Refractory giardiasis in Spanish travellers. Travel Med Infect Dis. 2013; 11(2): 126-9.

20. Iza JA, Iza SN, Olivera MJ. Giardiasis: report of a case refractory to treatment. Clin Microbiol Infect. 2018; 24(1): 37-42.

21. Campanati L, Troester H, Monteiro-Leal LH, Spring H, Trendelenburg MF, de Souza W. Tubulin diversity in trophozoites of Giardia lamblia. Histochem Cell Biol. 2003; 119: 323-31.

22. Nohynková E, Tumová P, Kulda J. Cell division of Giardia intestinalis: flagellar developmental cycle involves transformation and exchange of flagella between mastigonts of a diplomonad cell. Eukaryot Cell. 2006; 5: 753-61.

23. Mariante RM, Vancini RG, Melo AL, Benchimol M. Giardia lamblia: Evaluation of the in vitro effects of nocodazole and colchicine on trophozoites. Exp Parasitol. 2005; 110(1): 62-72.

24. Weber K, Schneider A, Westermann S, Müller N, Plessmann U. Posttranslational modifications of alpha- and beta-tubulin in Giardia lamblia, an ancient eukaryote. FEBS Lett. 1997; 419: 87-91.

25. Pérez-Rangel A, Hernández JM, Castillo-Romero A, Yépez-Mulia L, Castillo R, Hernández-Luis F, et al. Albendazole and its derivative JVG9 induce encystation on Giardia intestinalis trophozoites. Parasitol Res. 2013; 112(9): 3251-7.

26. Weiland ME, McArthur AG, Morrison HG, Sogin ML, Svärd SG. Annexin-like alpha giardins: a new cytoskeletal gene family in Giardia lamblia. Int J Parasitol. 2005; 35: 617-26.

27. Kim J, Lee HY, Lee MA, Yong TS, Lee KH, Park SJ. Identification of $\alpha-11$ giardin as a flagellar and surface component of Giardia lamblia. Exp Parasitol. 2013; 135(2): 227-33.

28. Stefanic S, Palm D, Svärd SG, Hehl AB. Organelle proteomics reveals cargo maturation mechanisms associated with Golgi-like encystation vesicles in the early-diverged protozoan Giardia lamblia. J Biol Chem. 2006; 281: 7595-7604.

29. Paredez AR, Assaf ZJ, Sept D, Timofejeva L, Dawson SC, Wang $\mathrm{CJ}$, et al. An actin cytoskeleton with evolutionarily conserved functions in the absence of canonical actin-binding proteins. Proc Natl Acad Sci USA. 2011; 108: 6151-6.

30. Castillo-Romero A, León-Ávila G, Wang CC, Pérez-Rangel A, Camacho-Nuez M, García-Tovar C, et al. Rab11 and actin cytoskeleton participate in Giardia lamblia encystation, guiding the specific vesicles to the cyst wall. PLoS Negl Trop Dis. 2010; 6: 1-14. 